\title{
Informed spatial basis functions in minimum norm solutions for the electromagnetic source localisation problem.
}

\author{
Phillips C. ${ }^{1,2,3}$, Rugg M.D. ${ }^{2}$, Friston K.J. ${ }^{1}$ \\ ${ }^{1}$ Wellcome Department of Cognitive Neurology, Institute of Neurology, UCL, London, UK ; \\ ${ }^{2}$ Institute of Cognitive Neuroscience, UCL, London, UK; \\ ${ }^{3}$ Cyclotron Research Centre, University of Liège, Liège, Belgium
}

\section{Introduction}

Linear inverse solutions have been applied extensively to solve the bioelectromagnetic inverse problem. In contrast to discrete dipole models, linear inverse solutions do not require any assumptions about the number of active sources and lead to a fully $3 \mathrm{D}$ representation of the electrical activity of the brain. However, the problem is underdetermined: there are many more parameters to estimate (relative to the number of dipole locations considered) than data available (relative to the number of electrodes). In order to ensure the uniqueness of the solution, existing linear methods generally apply constraints on the solution, for example: minimum 2-norm, maximum smoothness [1], optimal resolution [2], etc. These methods provide solutions with relatively poor spatial resolution because they neglect, wholly or in part, anatomical information relevant to the real source distribution.

Our method aims to model the spatial source distribution by using a set of basis functions. By appropriately defining these basis functions, we are able to include a priori information about the sources and our solutions will de facto belong to the subspace spanned by these basis functions. The priors enter as constraints on the covariance structure of the source power (over space), and are used to motivate the selection of a spatial basis set that maximises the information between the sources and their projection on that set. The orientation of each dipole is fixed and orthogonal to the cortical sheet, and therefore only the amplitude of the sources remains unknown. In a second step, we solve for the source distribution using a "classical" minimum norm method. Other methods could also be applied as the informed basis functions are generated before the computation of the solution.

Here we test our method using a realistic head model and noiseless instantaneous simulated data.

\section{Method}

The sources of the EEG signal are, in the absence of any data, assumed to conform to a fixed, uniform, three-dimensional distribution of current dipoles throughout the entire brain volume. Three strong assumptions are made about the sources: they are located in the grey matter, they are oriented orthogonal to the cortical sheet and, for a sufficiently dense dipole distri- bution, they present some locally coherent activity (or smooth activity along the cortical sheet) [3]. By fixing the orientation of each dipole, we can reduce the source localisation problem from a vectorial problem into a scalar problem. The two other assumptions, spatial smoothness and localisation within grey matter, are used to calculate the basis functions set.

The matrix $K$ represents the smoothness along the cortical sheet. A current dipole represents the coherent electrical activity of the brain over a small volume. A grey/white matter coefficient is determined for each dipole such that its value is: 0 if the volume around the dipole contains only white matter, 1 if the volume contains only grey matter and a value between 0 and 1 otherwise. These coefficients are contained in the diagonal matrix $G$.

If other functional imaging results (e.g. the findings of a PET or fMRI study) are available, they may also help to solve the source localisation problem. This information has to be regarded as probabilistic [4] and therefore can be used to generate the basis functions set. It could also be introduced at the second step of the method, when the minimum norm solution is sought. These additional probabilistic constraints are either combined with $G$ (if used to generate the basis functions) or represented by the matrix $H$ (if introduced at the second step).

The source localisation problem can be stated as:

$$
v=L j
$$

where $v$ is the electric potential at the electrodes, $j$ the amplitude of each current dipole and $L$ is the lead field linking $j$ and $v$. The orientation of the current dipole is included in $L$ so that $j$ represents only the amplitude of the dipole.

To reduce the solution space $j$ to $k$, we want $j$ and $k$ to be related by:

$$
j=R k \Leftrightarrow R^{T} j=k
$$

where $R$ maximises the mutual information (MI) [5] between $j$ and $k$ :

$$
\mathrm{MI}(j, k)=\mathrm{H}(j)+\mathrm{H}(k)-\mathrm{H}(j \cap k)=\mathrm{H}(k)
$$

because $\mathrm{H}(j)=\mathrm{H}(j \cap k)$.

Information theory tells us that if $k$ is a multinormal system then: 


$$
\mathrm{H}(k) \propto \ln \left(\left|C_{k}\right|\right)=\sum \ln \lambda_{i}
$$

where $C_{k}=k k^{T}$ is the variance/covariance matrix of $k$ and $\lambda_{i}$ its eigenvalues.

We then obtain:

$$
\operatorname{MI}(j, k) \propto \ln \left|R^{T} j j^{T} R\right|=\sum \ln \lambda_{i}
$$

and the optimum $R$ should be chosen as the eigenvectors of $j j^{T}$ corresponding to the highest eigenvalues $\lambda_{i}$.

We are now able to include our prior knowledge, $K$ and $G$, about $j$ :

- Prior 1, smoothness $K: j j^{T}=K^{T} K$ (correlation matrix).

- Prior 2, grey/white matter $G$ :

$$
j j^{T}=G^{T / 2} K^{T} K G^{1 / 2} \text {. }
$$

Finally $R$ is obtained by calculating the eigenvalue decomposition of $G^{T / 2} K^{T} K G^{1 / 2}$ or preferably by using a singular value decomposition:

$$
U S V^{T}=\operatorname{svd}\left(K G^{1 / 2}\right)
$$

and keeping the highest singular values in $S$, see fig. 1 and the corresponding vectors in $V$.

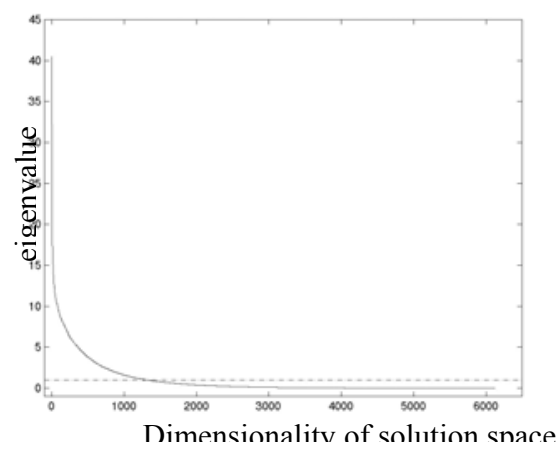

Figure 1, decreasing eigenvalues of $G^{T / 2} K^{T} K G^{1 / 2}$

We can now reformulate the source localisation problem like this:

$$
v=L_{R} k
$$

where $L_{R}=L R$ and $k=R^{T} j$. To solve this latter problem we use a minimum norm method:

$$
k=\arg \min _{k}\left\{\left\|L_{R} k-v\right\|^{2}+\lambda\|H R k\|^{2}\right\}
$$

We have assumed noiseless data, so the solution will be:

$$
k=T_{R} v \text { or } j=T v
$$

where $T=R T_{R}$ and

$$
T_{R}=\left(R^{T} H^{T} R H\right)^{-1} L_{R}^{T}\left[L_{R}\left(R^{T} H^{T} R H\right)^{-1} L_{R}^{T}\right]^{-1}
$$

\section{Application}

We have applied the method described above using a realistic head model generated from structural MRI data. The lead field matrix $L$, the solution of the "forward problem", is calculated with the "boundary element method" [6] for a set of 61 quasi equidistant electrodes (see fig. 2) and 6122 dipoles spread within the brain volume on a regular three dimensional grid. The cortical surface is extracted for each hemisphere using the "BrainVoyager" software package [7], see fig. 3 .

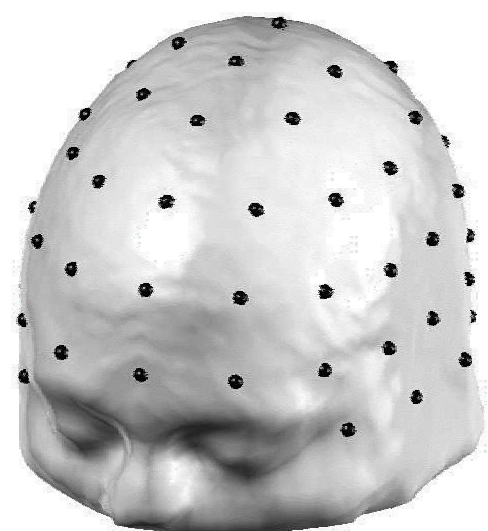

Figure 2, scalp surface with electrode locations

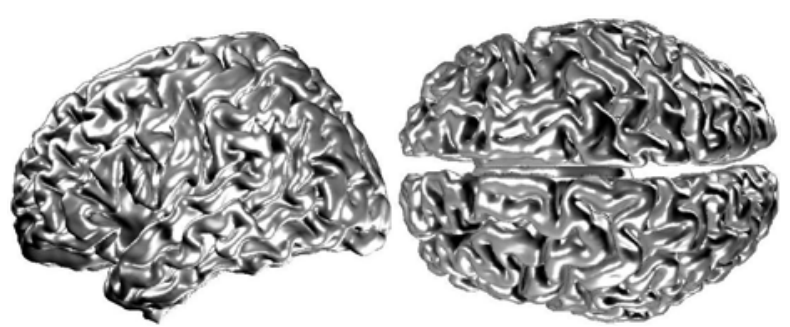

Figure 3, views of the cortical surface

The orientation of each dipole is defined to be normal to the closest cortical sheet patch, as shown in fig. 4, and is included in the lead field $L$.
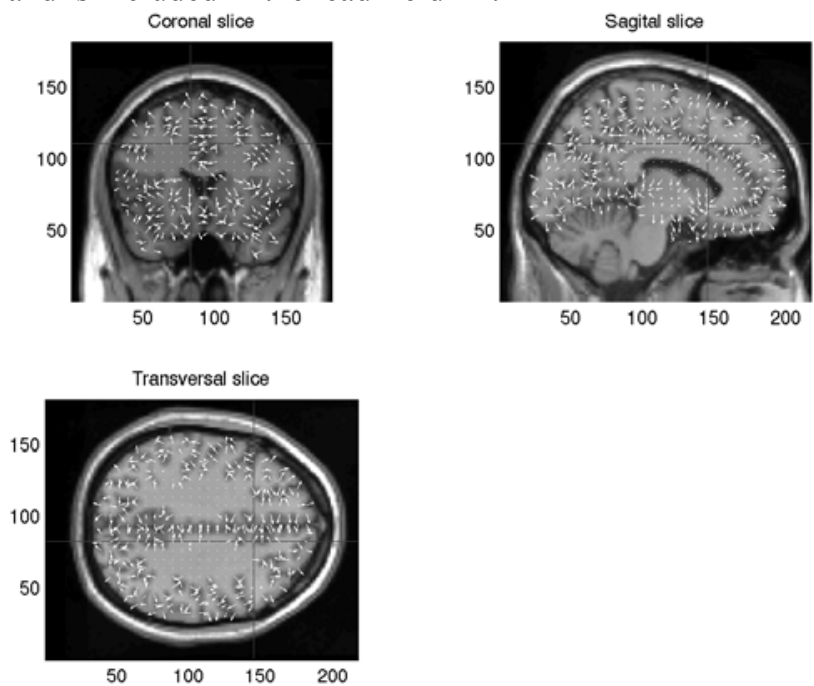

Figure 4, oriented dipoles in the grey matter.

We segment the MRI with the segmentation function of the SPM software package [8], and the grey matter image is averaged over each dipole cubic volume to 
provide the grey/white matter coefficient of the matrix $G$, see fig. 5. To estimate the smoothness over the cortical sheet expressed by the matrix $K$, we use Dijkstra's algorithm [9] to measure the distance between any two points (here the closest cortical points to the dipoles) on a tessellated irregular surface.

With $K$ and $G$ we calculate the singular value decomposition $U S V^{T}=\operatorname{svd}\left(K G^{1 / 2}\right)$ and keep only the singular vectors of $V$ corresponding to the normalised singular values greater than unity (see fig. 1). Eventually, from a solution space $j$ of 6122 dimensions, we end up with a reduced solution space $k$ of 1562 dimensions (a reduction of almost 85 percent), and the same number of orthonormal basis functions in $R$. Thereafter we can simply calculate the inverse solutions: $T_{R}$ and $T$. We will refer to this solution as the "informed basis functions" (IBF) solution.

We also calculate a direct minimum norm $(\mathrm{MN})$ solution without reducing a priori the solution space.

\section{Results and discussion}

We simulated sources that might be active in a typical cognitive ERP study, by assuming that the task activated left and right occipital cortex, left and right fusiform regions and a right frontal area. We define only one active dipole in each occipital region. For the left and right fusiform and the frontal regions we modelled distributed sources. Figure 6 shows the sources configuration. We used these sources to generate the EEG signal at each electrode and also to produce a simulated fMRI activation map, shown in fig. 7.

We first consider both MN and IBF solutions without the fMRI activation map, setting the matrix $H$ to be the identity matrix. We reconstruct the 2 occipital sources as if they were simultaneously active, then the 2 fusiform sources together and finally the single frontal source, see fig. 8. Both method localise correctly the superficial sources (occipital and frontal) but fail to reconstruct the deep fusiform sources. Because smoothness is incorporated to constrain the basis functions, the IBF solution is smoother and more distributed than the MN solution. This latter solution tends to concentrate all the activity on a few superficial voxels. We then use the simulated fMRI a priori to constraint the solution of the MN approach and at the second step for the IBF approach. Similarly, we reconstruct the 2 occipital sources as if they were simultaneously active, then the 2 fusiform sources together and finally the single frontal source, see fig. 9. Here all the sources are reconstructed accurately, including the deep fusiform sources.

In this preliminary work, therefore we have only used noise free simulated data. Under these optimal conditions, there is no need for much constraint and both $\mathrm{MN}$ and IBF methods yielded reasonable 3D solutions. The aim of the IBF method however is to allow the inclusion of any constraint on the solution. For exam- ple, further developments will incorporate temporal basis function [10]. These (with the spatial basis functions presented here) should be useful in the treatment of more realistic data that is time extended data mixed with real noise and background activity. In this case, spatio-temporal constraints are crucial to reconstruct even superficial sources.

\section{Conclusions}

We have introduced an original and general framework for solving the EEG source localisation problem that allows the inclusion of any a priori knowledge about the solution space. We have successfully applied our method on a spatially realistic model and data with a minimum norm approach. We tested our method on noiseless instantaneous simulated data. The results obtained show good spatial resolution and robust performance.

We plan to extend our method to more realistic, time extended data mixed with real noise and background EEG activity.

\section{References}

[1] Pascual-Marqui, R.D., Michel, C. and Lehman, D., Low-resolution electromagnetic tomography: a new method for localising electrical activity in the brain, Int. J. Psychophysiology, 1994, 18: 49-65.

[2] Grave de Peralta, R., Hauk, O., Gonzalez Andino, S., Vogt H and Michel C, Linear inverse solutions with optimal resolution kernels applied to electromagnetic tomography, Human brain mapping, 1997, 5: 454-467.

[3] Dale, A.M., Sereno, M.I., Improved localisation of cortical activity by combining EEG and MEG with MRI cortical surface reconstruction: a linear approach, J. Cogn. Neurosci., 1993, 5:162-176.

[4] Rugg, M.D., Convergent approaches to electrophysiological and haemodynamic investigations of memory, Hum. Brain Mapp., 1998, 6:394-398.

[5] Jones, D.S., Elementary information theory, Oxford, Carendon Press, 1979.

[6] Sarvas, J., Basic mathematical and electromagnetic concepts of the biomagnetic inverse problem, Phys. Med. Biol., 1987, 32: 11-22.

[7] Goebel, R., Brain Innovation Rainer Goebel, http://www.BrainVoyager.de.

[8] Ashburner, J. and Friston, K.J, Multimodal image coregistration and partitioning - a unified framework, NeuroImage, 1997, 6:209-217.

[9] Dijkstra, E.W., A note on two problems in connection with graphs, Numerische Mathematik, 1959, 1; 269-271. 
[10] Phillips, C., Rugg, M.D. and Friston, K.J., A priori spatio-temporal basis functions in minimum norm solutions, HBM99 abstract, to be published in NeuroImage.

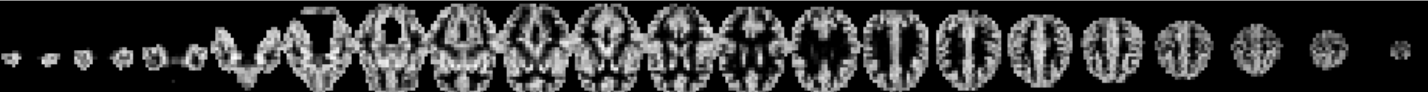

Figure 5, grey/white matter value susbampled for the dipoles location.

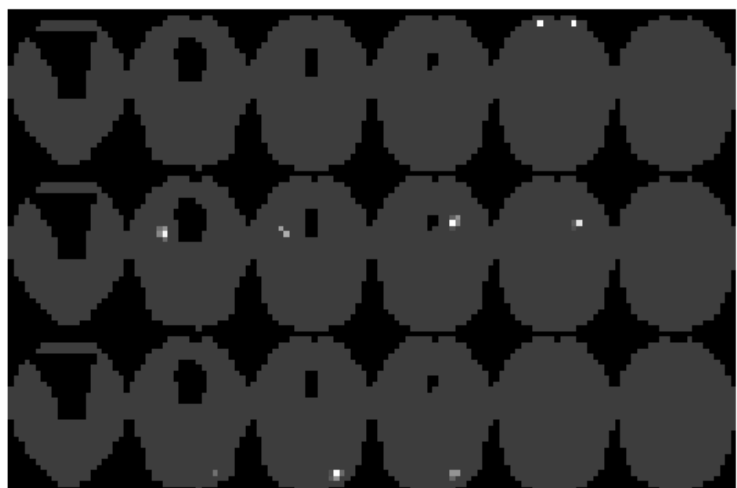

Figure 6, active sources: from top to bottom, occipital, fusiform and frontal sources.

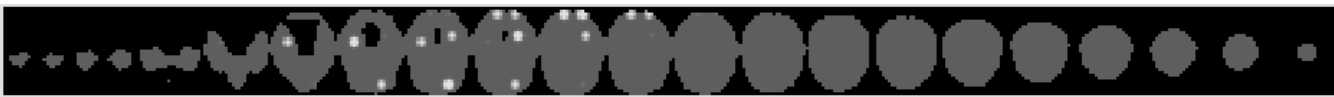

Figure 7, a priori fMRI statistical map.
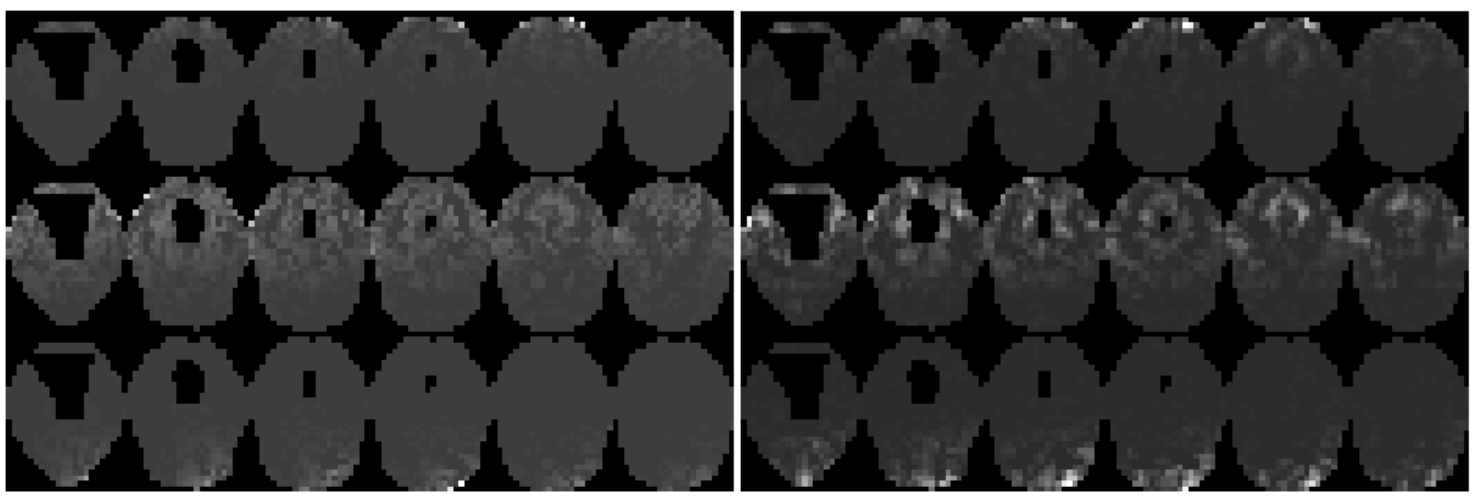

Figure 8, reconstruction of the sources without functional imaging a priori, left: simple minimum norm, right: informed basis functions and minimum norm; from top to bottom: occipital, fusiform and frontal sources.
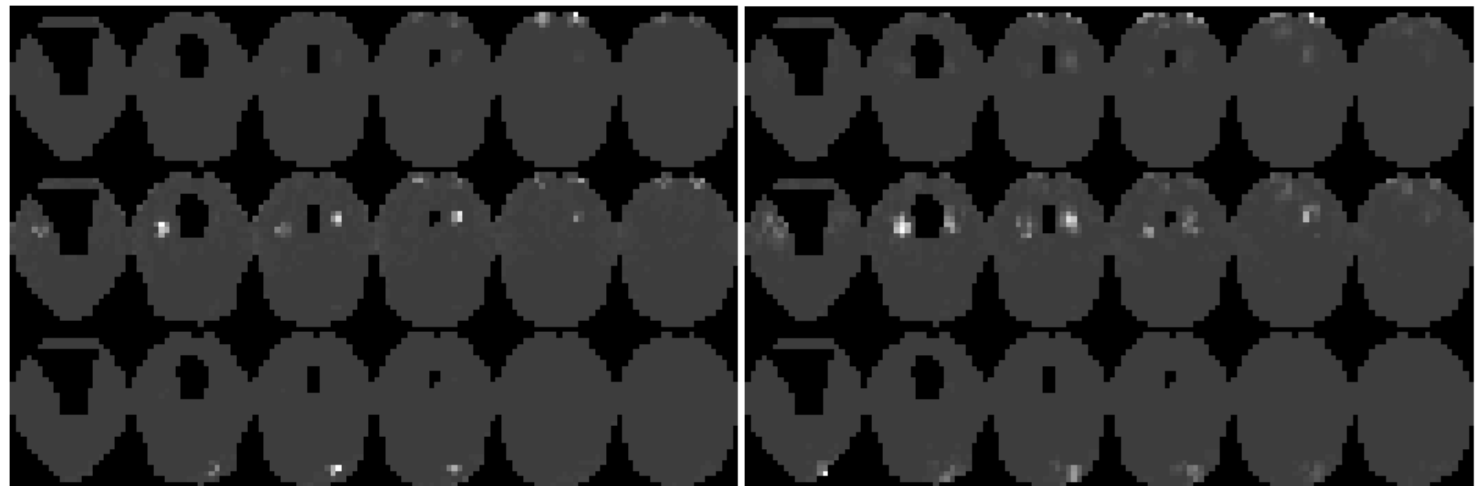

Figure 9, reconstruction of the sources with functional imaging a priori, left: simple minimum norm, right: informed basis functions and minimum norm; from top to bottom: occipital, fusiform and frontal sources. 